TECHNICAL NOTE

\author{
G.S. Young \\ K. Setayesh
}

\section{Spin-Echo Echo-Planar Perfusion MR Imaging in the Differential Diagnosis of Solitary Enhancing Brain Lesions: Distinguishing Solitary Metastases from Primary Glioma}

SUMMARY: Unlike the more widely reported gradient-echo echo-planar perfusion-weighted imaging (EPI-PWI) technique, spin-echo (SE) EPI relative cerebral blood volume maps select for blood volume in microvessels $<8 \mu \mathrm{m}$ in diameter. This first report of SE-EPI PWI for distinguishing brain metastasis from high-grade glioma demonstrated $88 \%$ sensitivity and $72 \%$ specificity in 83 patients. We discuss differences in microvessel architecture between high-grade glioma and brain metastasis that may explain the surprising success of SE-EPI in this application and may deserve further investigation.
D istinction of brain metastasis from high-grade glioma by MR imaging remains an important unsolved clinical problem. Percentage recovery analysis of time-intensity curves from gradient-echo echo-planar (GE-EPI) dynamic susceptibility contrast perfusion-weighted imaging (PWI ${ }^{1}$ has been proposed to distinguish these tumor types by assessing increased capillary permeability in brain metastasis, but to our knowledge, no published report yet documents the accuracy of this method. Reports from the pathology literature suggest that in addition to permeability, brain metastasis and high-grade glioma neovessels differ substantially in vascular architecture, $^{2-7}$ and in particular, metastases may contain a substantially larger proportion of intermediate-sized dysplastic vessels up to $70 \mu \mathrm{m}$ in diameter. ${ }^{8-12}$ Recent literature demonstrates that spin-echo EPI (SE-EPI) PWI has a substantially different vessel-size sensitivity profile from GE-EPI PWI. ${ }^{13-15}$ Because SE-EPI PWI relative cerebral blood volume (rCBV) maps reflect nearly exclusively the blood volume of microvessels $<8 \mu \mathrm{m}$ in diameter, in contrast to the admixture of microvessels and larger vessels assessed with GE-EPI, we hypothesized that SE-EPI rCBV maps would allow reliable distinction of brain metastasis from high-grade glioma. To our knowledge, this is the first report using SE-EPI PWI to make this distinction.

\section{Technique}

Forty-three subjects with high-grade glioma (20 cases of glioblastoma multiforme, 14 anaplastic astrocytomas, 7 anaplastic oligoastrocytomas, and 2 unspecified high-grade gliomas) and 40 patients with brain metastasis ( 2 sarcomas; 38 carcinomas, including 20 lung, 5 melanoma, 1 basal cell, 4 breast, 1 esophageal, 2 colorectal, 1 prostate; and 4 adenocarcinomas of unknown primary) were included chronologically from our institutional data base. Ninety-eight percent (42) of patients with high-grade glioma and 68\% (27) of patients with brain metastasis had received previous chemotherapy; 63\% (27) of patients with high-grade glioma and $85 \%$ of patients with metastasis (34) had

Received April 10, 2008; accepted after revision June 5.

From the Department of Radiology of Brigham and Women's Hospital, Harvard Medical School, Boston, Mass.

Both authors contributed equally to this work

Please address correspondence to Geoffrey S. Young, MD, Brigham and Women's Hospital, Radiology Department, 75 Francis St, Boston, MA 02115; e-mail: GSYOUNG@PARTNERS. ORG

DOI 10.3174/ajnr.A1239 received radiation (Table 1). Following institutional protocol, SE-EPI PWI $(\mathrm{TE}=80 \mathrm{~ms}, \mathrm{TR}=1900-2216.7 \mathrm{~ms}$, section thickness $=10$ $\mathrm{mm}$, matrix $=128^{2}-256^{2}$ ) was performed by using a $4-\mathrm{mL} / \mathrm{s}$ bolus injection of 30-mL double-dose gadopentetate dimeglumine (Magnevist; Bayer HealthCare Pharmaceuticals, Wayne, NJ) (0.15-0.3 $\mathrm{mmol} / \mathrm{kg}$ depending on body weight) and was coregistered with delayed postgadolinium T1-weighted imaging. Imaging was performed on $1.5 \mathrm{~T}$ or $3 \mathrm{~T}$ commercial MR imaging scanners (GE Healthcare, Milwaukee, Wis), and PWI data were processed by using a perfusionprocessing software package (GADW 4.3 FuncTool software, GE Healthcare), which creates color rCBV maps by calculating the change in transverse relaxivity (R2) from signal-intensity measurements, integrates the area under the relaxivity curve and corrects for $\mathrm{TE}$, according to the following standard formula:

$$
r C B V=\sum_{i=0}^{N} \Delta R 2_{t}=\frac{-1}{T E} \sum_{i=0}^{N} \ln \left(\frac{S_{i}}{S_{0}}\right) .
$$

Following previously published "hot spot" methods, we selected a roughly $100-\mathrm{mm}^{2}$ region of interest (ROI) within the region of maximal $\mathrm{rCBV}$ and recorded the average $\mathrm{rCBV}$ within the ROI as the rCBVmax. ${ }^{16,17}$ We calculated a normalized rCBV (nCBV) by dividing rCBVmax by the rCBV of an equivalent ROI in the contralateral normal-appearing white matter ${ }^{16}$ and performed percentage recovery analysis in the same ROI according to previously reported GE-EPI techniques. $^{1}$

\section{Results}

The average nCBV within enhancing high-grade glioma was $1.53 \pm 0.79(0.59-4.05)$ compared with $0.82 \pm 0.40$ for brain metastasis $(0.48-2.12)$, and differences between $\mathrm{nCBV}$ averages and ranges for the 2 groups were significant $(P<.05)$ (Fig $1 C)$. Sensitivity and specificity for detection of brain metastasis were $95 \%$ and $51 \%$, respectively, by using an nCBV threshold of 1.3 ; and $36 \%$ and $93 \%$, by using a 0.7 threshold. The optimal threshold occurred at 1.0, where sensitivity and specificity were $88 \%$ and $72 \%$ (Table 2 ).

Percentage recovery analysis yielded average signal-intensity recoveries of 0.86 for high-grade glioma and 0.75 for brain metastasis $(P<.05)$. Percentage recovery analysis sensitivity and specificity to distinguish brain metastasis from high-grade glioma were $18 \%$ and $93 \%$ by using a previously reported ${ }^{1}$ signal-intensity recovery threshold of 0.66 . Optimal accuracy, 


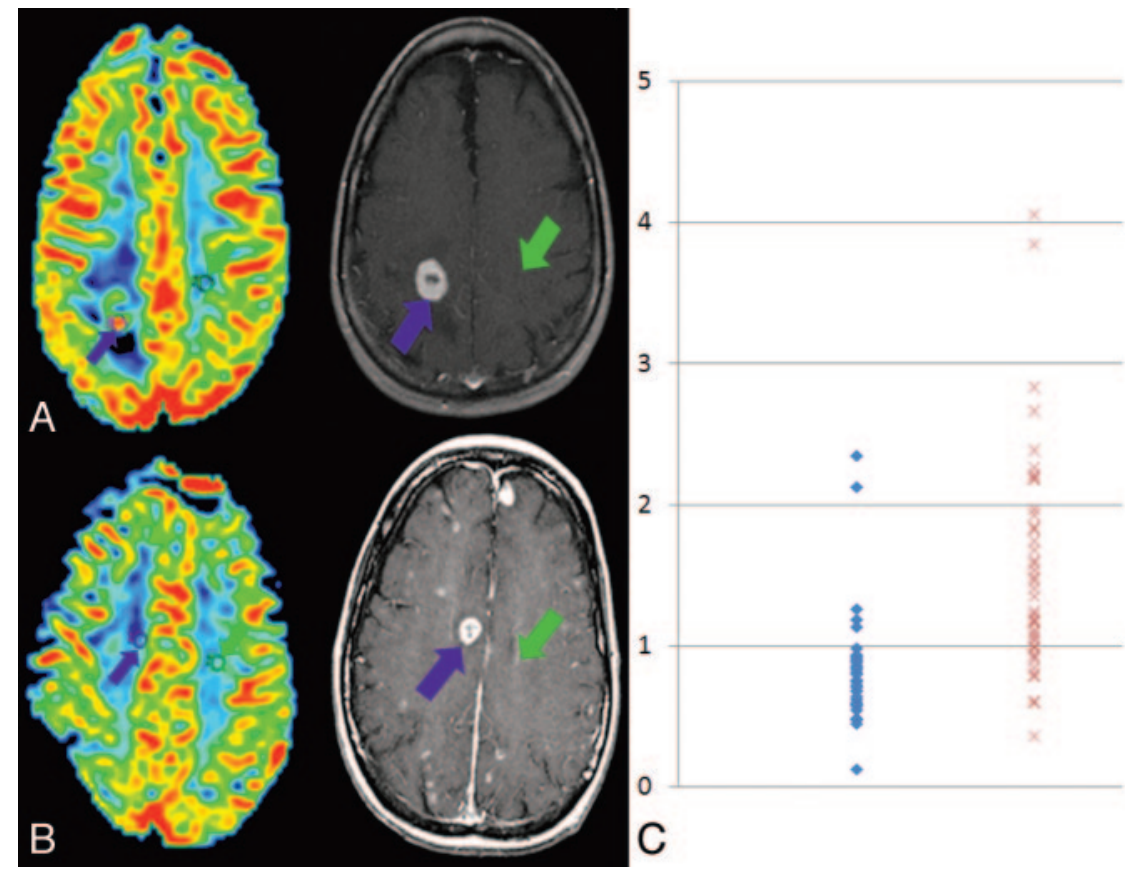

Fig 1. $A$ and $B$, rCBV maps (left) demonstrate increased blood volume in the enhancing high-grade glioma $(A)$ and decreased blood volume in the enhancing brain metastasis (B). Corresponding T1-weighted images are shown (center) with black arrows representing the location of ROI selection in the lesion and white arrows indicating control ROI location in normal appearing contralateral white matter placement. C, Scatterplot of the nCBV measurements in brain metastasis (blue diamonds) and high-grade glioma (red squares). (Color figure is available on-line only at www.ajnr.org.)

\begin{tabular}{lccll}
\hline \multicolumn{4}{l}{ Table 1: Patient Demographics } & \\
\hline & Age & Sex & $\begin{array}{c}\text { Previous } \\
\text { Chemotherapy }\end{array}$ & $\begin{array}{c}\text { Previous } \\
\text { Radiotherapy }\end{array}$ \\
\hline Glioma & $49.9 \pm 11.21$ & $21 \mathrm{~F} / 22 \mathrm{M}$ & $98 \%(20 \mathrm{~F} / 22 \mathrm{M})$ & $63 \%(6 \mathrm{~F} / 10 \mathrm{M})$ \\
Metastases & $56.5 \pm 11.60$ & $18 \mathrm{~F} / 22 \mathrm{M}$ & $68 \%(6 \mathrm{~F} / 7 \mathrm{M})$ & $85 \%(2 \mathrm{~F} / 4 \mathrm{M})$ \\
Overall & $53.1 \pm 11.80$ & $39 \mathrm{~F} / 44 \mathrm{M}$ & $83 \%(7 \mathrm{~F} / 7 \mathrm{M})$ & $73 \%(8 \mathrm{~F} / 14 \mathrm{M})$
\end{tabular}

Note:- F indicates female; $M$, male.

\begin{tabular}{lcc}
\hline $\begin{array}{l}\text { Table 2: Diagnostic Accuracy of SE-EPI } \mathbf{n C B V} \text { vs. Percent Recovery } \\
\text { Using } \mathbf{0 p t i m a l} \text { Thresholds of } \mathbf{n C B V}=\mathbf{1 . 0} \text { and } \% \text { recovery } \mathbf{=} \mathbf{6 6} \%\end{array}$ \\
\hline & nCBV & $\begin{array}{c}\text { Percentage } \\
\text { Recovery }\end{array}$ \\
\hline Glioma & $1.52 \pm 0.78$ & $0.85 \pm 0.25$ \\
Metastases & $0.82 \pm 0.39$ & $0.75 \pm 0.15$ \\
Sensitivity & 0.87 & 0.18 \\
Specificity & 0.72 & 0.93 \\
\hline
\end{tabular}

Note:-nCBV indicates normalized cerebral blood volume.

obtained by using a signal-intensity recovery threshold of 0.85 , yielded a sensitivity and specificity of $84 \%$ and $48 \%$ (Table 2).

\section{Discussion}

In this retrospective series, SE-EPI-derived rCBV maps allowed reliable distinction of brain metastasis from highgrade glioma and were superior to percentage recovery analysis for distinguishing brain metastasis from highgrade glioma by using SE-EPI (Fig 1). Because of the limitations regarding the retrospective design of this initial report, further study is required before this finding can be applied clinically. Most important, although both groups received radiation and chemotherapy, differences in treatment response between the groups could conceivably contribute to the differences observed.

Although definitive testing of our hypothesis that the success of SE-EPI is related to differences in vessel-size distribution between brain metastasis and high-grade glioma is beyond the scope of this report, a number of limitations of the current data deserve consideration in the design of future studies. First, as our percentage recovery analysis demonstrates, there is a significant difference between the brain metastasis and high-grade glioma groups in microvessel permeability. Future studies designed to investigate whether the observed effect is actually related to vesselsize differences between brain metastasis and high-grade glioma will require more rigorous correction for T1 shortening effects due to first-pass capillary leak. ${ }^{18}$ Additionally, the use of interleaved GE-SE vessel-size imaging ${ }^{5,6}$ may be of interest. Finally, because of the paucity of direct analyses of metastatic vessel-size distribution in the pathology literature, correlation with microscopic imaging would be valuable.

\section{Conclusion}

SE-EPI-derived rCBV maps promise to assist in accurate preoperative distinction of brain metastasis from high-grade glioma. If more rigorous analysis in treatment-naïve patients and prospective investigation support the preliminary findings presented here, SE-EPI PWI may prove an important clinical tool.

\section{References}

1. Cha S, Johnson G, Wadghiri YZ, et al. Dynamic, contrast-enhanced perfusion MRI in mouse gliomas: correlation with histopathology. Magn Reson Med 2003;49:848-55

2. Deane BR, Lantos PL. The vasculature of experimental brain tumors. Part 1. A sequential light and electron microscope study of angiogenesis J Neurol Sci 1981;49:55-66

3. Dennie J, Mandeville JB, Boxerman JL, et al. NMR imaging of changes in vascular morphology due to tumor angiogenesis. Magn Reson Med 1998;40: 793-99 
4. Kremer S, Grand S, Berger F, et al. Dynamic contrast-enhanced MRI: differentiating melanoma and renal carcinoma metastases from high-grade astrocytomas and other metastases. Neuroradiology 2003;45:44-49

5. Kiselev VG, Strecker R, Ziyeh S, et al. Vessel size imaging in humans. Magn Reson Med 2005;53:553-63

6. Tropres I, Grimault S, Vaeth A, et al. Vessel size imaging. Magn Reson Med 2001;45:397-408

7. Aronen HJ, Gazit IE, Louis DN, et al. Cerebral blood volume maps of gliomas: comparison with tumor grade and histologic findings. Radiology 1994;191: 41-51

8. Nussbaum E, Djalilian H, Cho K, et al. Brain metastases: histology, multiplicity, surgery, and survival. Cancer 1996;78:1781-88

9. Lu W, Bucana CD, Schroit AJ. Pathogenesis and vascular integrity of breast cancer brain metastasis. Int J Cancer 2007;120:1023-26

10. Onodera H, Nagayama S, Tachibana $T$, et al. Brain metastasis from colorectal cancer. Int J Colorectal Dis 2005;20:57-61

11. McCurley CR, Shivers RR, Del Maestro RF. Quantitative comparison of the morphology of the microvasculature of primary lung lesions and metastatic brain tumors. J Submicrosc Cytol Pathol 1998;30:257-69

12. Sangiorgi S, Congiu T, Manelli A, et al. The three-dimensional microvascular architecture of the human Kaposi sarcoma implanted in nude mice: A SEM corrosion casting study. Microvasc Res 2006;72:128-35. Epub 2006 Aug 22

13. Donahue KM, Krouwer HGJ, Rand SD, et al. Utility of simultaneously acquired gradient-echo and spin-echo cerebral blood volume and morphology maps in brain tumor patients. Magn Reson Med 2000;43:845-53

14. Sugahara T, Korogi Y, Kochi M, et al. Perfusion-sensitive MR imaging of gliomas: comparison between gradient-echo and spin-echo echo-planar imaging techniques. AJNR Am J Neuroradiol 2001;22:1306-15

15. Speck O, Chang L, DeSilva NM, et al. Perfusion MRI of the human brain with dynamic susceptibility contrast: gradient-echo versus spin-echo techniques. $J$ Magn Reson Imaging 2000;12:381-87

16. Calli C, Kitis O, Yunten N, et al. Perfusion and diffusion MR imaging in enhancing malignant cerebral tumors. Eur J Radiol 2006;58:394-403

17. Cha S, Tihan T, Crawford F, et al. Differentiation of low-grade oligodendrogliomas from low-grade astrocytomas by using quantitative blood-volume measurements derived from dynamic susceptibility contrast-enhanced MR imaging. AJNR Am J Neuroradiol 2005;26:266-73

18. Boxerman JL, Schmainda KM, Weiskoff RM. Relative cerebral blood volume maps corrected for contrast agent extravasation significantly correlate with glioma tumor grade, whereas uncorrected maps do not. AJNR Am J Neuroradiol 2006;27:859-67 